Ti re- dependent var i at i onal pri nci pl e wi th const $r$ ai nt $s$ for par anet $r$ i zed wave f unct i ons

\begin{tabular}{|l|l|}
\hline 著者 & OHTA Kat suhi sa \\
\hline $\begin{array}{l}\text { j our nal or } \\
\text { publ i cat i on ti tl e }\end{array}$ & $\begin{array}{l}\text { Physi cal revi ew. Ser. 3. A, At om c, nol ecul ar, } \\
\text { and opt i cal physi cs }\end{array}$ \\
\hline vol une & 70 \\
\hline number & 2 \\
\hline page range & 22503-1- 22503-9 \\
\hline year & 2004 08-09 \\
\hline URL & ht t p: //hdl . handl e. net /10258/203 \\
\hline
\end{tabular}


Ti re- dependent var i at i onal pri nci pl e wi th const $r$ ai nt $s$ for par anet $r$ i zed wave f unct i ons

\begin{tabular}{|l|l|}
\hline 著者 & OHTA Kat suhi sa \\
\hline $\begin{array}{l}\text { j our nal or } \\
\text { publ i cat i on ti tl e }\end{array}$ & $\begin{array}{l}\text { Physi cal revi ew. Ser. 3. A, At om c, nol ecul ar, } \\
\text { and opt i cal physi cs }\end{array}$ \\
\hline vol une & 70 \\
\hline number & 2 \\
\hline page range & 22503-1- 22503-9 \\
\hline year & 2004 08-09 \\
\hline URL & ht t p: //hdl . handl e. net /10258/203 \\
\hline
\end{tabular}




\title{
Time-dependent variational principle with constraints for parametrized wave functions
}

\author{
Katsuhisa Ohta* \\ Department of Applied Chemistry, Muroran Institute of Technology, Muroran, Hokkaido 050-8585, Japan
}

(Received 8 January 2004; published 9 August 2004)

\begin{abstract}
The time-dependent variational principle in the stationary action principle form is formulated with constraint conditions for parametrized wave functions. The constraint conditions are classified into the first and the second classes as in Dirac's constrained classical mechanics based on the commutability of operators. If the local bases in the parametrized wave functions construct a complete basis set, the classification of constraints with the commutator becomes equivalent to that with the complex generalized Poisson brackets (CGPB). However, in approximate wave functions with limited variational parameters, the equivalence between the CGPB and the commutator of operators is lost. The first-class constraints and the constants of motion classified with the commutator should be reconsidered as the pseudo-second-class constraints in the approximate wave functions.
\end{abstract}

DOI: 10.1103/PhysRevA.70.022503

PACS number(s): 31.15.Pf

\section{INTRODUCTION}

In many dynamical processes in physics and chemistry, the time-dependent picture enables an intuitive and vivid understanding of the processes. Particularly for electrons and nuclei in molecules, the time-dependent variational principle (TDVP) [1-6] has become one of the most popular methods to obtain the quantum-mechanical time-dependent wave functions. Recent theoretical works have been reviewed in Ref. [7]. From the viewpoint of the variational method, however, only the normalization condition for wave functions has been considered as the constraint in the TDVP in spite of various techniques in the variational method $[8,9]$. In our previous work [10], the TDVP has been formulated with not only the normalization but also general constraint conditions for wave functions to extend the applicability of the TDVP, e.g., to quantum dynamics in subspaces which are specified by some physical or artificial constraints. The constraint conditions have been classified into the first and the second classes according to the terminology of Dirac's constrained classical mechanics [11,12].

In this work, we formulate the TDVP with constraints by introducing explicit variational parameters of wave functions. In many investigations to use the TDVP, various parametrizations of wave functions have been considered as in the time-dependent Hartree-Fock wave functions, Gaussian wave packets, and so on [7]. We adopt here a complex analytic parametrization of wave functions to keep the variational independence between $\delta \Psi$ and $\delta \Psi^{*}[4,13]$. The Euler equations in the parametrized TDVP are written in the form of the pseudo-classical-mechanics [13] where the equations of motion (EOM) are described by complex generalized Poisson brackets (CGPB). All the rich apparatus developed for the study of classical mechanics can be applied to the study of this motion. Unfortunately, however, in approximate wave functions constructed with limited variational parameters, the commutability between operators is not always

\footnotetext{
*Email address: ohta@mmm.muroran-it.ac.jp
}

kept in the CGPB. The classification of constraints to the first-class and the second-class constraints becomes not equivalent to that in exact wave functions [10]. Some constants of motion are also lost in the approximate wave functions. For example, even the norm of the wave functions, which is a typical first-class quantity, is not always conserved, although the expectation values of physical quantities may be obtained correctly by dividing with the norm. We reconsider such constraints in the approximate wave functions as the pseudo-second-class constraints.

In Sec. II, we give the EOM for the TDVP parameters with constraints. The Lagrange multipliers for the secondclass constraints are determined. In Sec. III, we show that the complete local bases in the parametrized wave functions can afford the equivalence between the CGPB and the commutator of operators. The classification of constraints to the first and the second classes with the CGPB is exactly the same as that with the commutator. In Sec. IV, we discuss approximate wave functions with limited variational parameters where the local bases are incomplete. A brief summary is given in Sec. V.

\section{TIME-DEPENDENT VARIATIONAL PRINCIPLE WITH CONSTRAINTS}

\section{A. Equations of motion of variational parameters}

A complex analytic parametrization of wave functions is formulted to keep the variational independence between $\delta \Psi$ and $\delta \Psi^{*}[4,13]$. A set of time-dependent variational parameters $\left\{z_{i}(t), z_{i}^{*}(t)\right\}_{i=1, M}$ is introduced to the trial wave function as

$$
\begin{aligned}
& \Psi(z ; x)=\Psi\left(z_{1}, z_{2}, \ldots, z_{M} ; x\right), \\
& \frac{\partial \Psi(z ; x)}{\partial z_{i}^{*}}=0 \quad(i=1, \ldots, M) .
\end{aligned}
$$

The variation of the trial function is reduced to those of the variational parameters in the expanded form with the local bases $\left\{\partial \Psi / \partial z_{i}\right\}_{i=1, M}$ as 


$$
\delta \Psi=\sum_{i=1}^{M} \delta z_{i} \frac{\partial \Psi}{\partial z_{i}}
$$

where the "local bases" means that the vectors $\partial \Psi / \partial z_{i}$, as the bases in the Hilbert space, generally depend on the variational parameters $\left\{z_{i}(t)\right\}_{i=1, M}$ in nonlinear parametrizations. Here we consider the normalization constraint of the wave function,

$$
g_{0}\left(z, z^{*}\right)=\langle\Psi|\hat{1}| \Psi\rangle-1=\left\langle\Psi\left|\hat{1}-\langle\Psi \mid \Psi\rangle^{-1}\right| \Psi\right\rangle=0,
$$

and the independent constraints with the Hermitian operators $\hat{g}_{i}$,

$$
g_{i}\left(z, z^{*}\right)=\left\langle\Psi\left|\hat{g}_{i}\right| \Psi\right\rangle=0 \quad(i=1, \ldots, 2 L),
$$

where the number of the constraints in Eq. (4) should be even in order to avoid the singularity in the determination of their Lagrange multipliers [10], as will be discussed in Sec. II B. A real Lagrangian is defined as

$$
\begin{aligned}
L\left(z, z^{*}\right)= & \left\langle\Psi\left|\frac{i \hbar}{2}\left(\vec{\partial}_{t}-\overleftarrow{\partial}_{t}\right)-\hat{H}\right| \Psi\right\rangle-\sum_{i=0}^{2 L} \lambda_{i} g_{i}\left(z, z^{*}\right), \\
= & \frac{i \hbar}{2} \sum_{i=1}^{M} \dot{z}_{i}\left\langle\Psi \mid \frac{\partial \Psi}{\partial z_{i}}\right\rangle-\frac{i \hbar}{2} \sum_{i=1}^{M} \dot{z}_{i}^{*}\left\langle\frac{\partial \Psi}{\partial z_{i}} \mid \Psi\right\rangle \\
& -\langle\Psi(z)|\hat{H}| \Psi(z)\rangle-\sum_{i=0}^{2 L} \lambda_{i} g_{i}\left(z, z^{*}\right),
\end{aligned}
$$

including the constraints with the Lagrange multipliers, $\lambda_{0}(t)$, and $\left\{\lambda_{i}(t)\right\}_{i=1,2 L}$. An action functional is also defined with the Lagrangian as

$$
\begin{aligned}
S\left[z, z^{*}\right]= & \int_{t_{0}}^{t_{1}} L\left(z, z^{*}\right) d t \\
= & \int_{t_{0}}^{t_{1}}\left[\frac{i \hbar}{2} \sum_{i=1}^{M} \dot{z}_{i}\left\langle\Psi \mid \frac{\partial \Psi}{\partial z_{i}}\right\rangle-\frac{i \hbar}{2} \sum_{i=1}^{M} \dot{z}_{i}^{*}\left\langle\frac{\partial \Psi}{\partial z_{i}} \mid \Psi\right\rangle\right. \\
& \left.-\langle\Psi(z)|\hat{H}| \Psi(z)\rangle-\sum_{i=0}^{2 L} \lambda_{i} g_{i}\left(z, z^{*}\right)\right] d t
\end{aligned}
$$

By requiring the action to be stationary $[6,10,13]$,

$$
\delta S\left[z, z^{*}\right]=\int_{t_{0}}^{t_{1}} \delta L\left(z, z^{*}\right) d t=0,
$$

with the fixed boundary conditions,

$$
\delta z_{i}^{*}\left(t_{0}\right)=\delta z_{i}^{*}\left(t_{1}\right)=\delta z_{i}\left(t_{0}\right)=\delta z_{i}\left(t_{1}\right)=0 \quad(i=1, \ldots, M),
$$

we obtain the equations of motion (EOM) for the TDVP parameters, $\left\{z_{i}(t), z_{i}^{*}(t)\right\}_{i=1, M}$, as

$$
i \hbar \sum_{j=1}^{M} \frac{d z_{j}}{d t}\left\langle\frac{\partial \Psi}{\partial z_{i}} \mid \frac{\partial \Psi}{\partial z_{j}}\right\rangle=\left\langle\frac{\partial \Psi}{\partial z_{i}}|\hat{H}| \Psi\right\rangle+\sum_{j=0}^{2 L} \lambda_{j} \frac{\partial g_{j}}{\partial z_{i}^{*}},
$$

$$
-i \hbar \sum_{j=1}^{M} \frac{d z_{j}^{*}}{d t}\left\langle\frac{\partial \Psi}{\partial z_{j}} \mid \frac{\partial \Psi}{\partial z_{i}}\right\rangle=\left\langle\Psi|\hat{H}| \frac{\partial \Psi}{\partial z_{i}}\right\rangle+\sum_{j=0}^{2 L} \lambda_{j} \frac{\partial g_{j}}{\partial z_{i}} .
$$

By introducing the new Hamiltonian operator with the constraints (4),

$$
\hat{H}^{\prime}=\hat{H}+\sum_{j=1}^{2 L} \lambda_{j} \hat{g}_{j}
$$

and the expectation value also with the normalization condition (3),

$$
K=\left\langle\Psi\left|\hat{H}^{\prime}+\lambda_{0} \hat{g}_{0}\right| \Psi\right\rangle=H^{\prime}+\lambda_{0} g_{0}=H+\sum_{j=1}^{2 L} \lambda_{j} g_{j}+\lambda_{0} g_{0},
$$

the EOM (9) and (10) are written simply in the matrix form as

$$
\left(\begin{array}{c}
\dot{\mathbf{z}} \\
\dot{\mathbf{z}}^{*}
\end{array}\right)=\frac{1}{i \hbar}\left(\begin{array}{cc}
0 & \mathbf{C}^{-1} \\
-\left(\mathbf{C}^{-1}\right)^{t} & 0
\end{array}\right)\left(\begin{array}{c}
\frac{\partial K}{\partial z} \\
\frac{\partial K}{\partial z^{*}}
\end{array}\right) .
$$

The Hermitian matrix $\mathbf{C}$ in the EOM (13) is the overlap matrix between the local bases,

$$
(\mathbf{C})_{i j}=\left\langle\frac{\partial \Psi}{\partial z_{i}} \mid \frac{\partial \Psi}{\partial z_{j}}\right\rangle \text {. }
$$

The time development of a function $F\left(z, z^{*}\right)$ is calculated with Eq. (13) as

$$
\begin{aligned}
\frac{d F(z, z *)}{d t} & =\sum_{i=1}^{M}\left[\dot{z}_{i}^{*} \frac{\partial F}{\partial z_{i}^{*}}+\dot{z}_{i} \frac{\partial F}{\partial z_{i}}\right] \\
& =\sum_{i=1}^{M} \sum_{j=1}^{M}\left[\frac{\partial F}{\partial z_{i}}\left(\mathbf{C}^{-1}\right)_{i j} \frac{\partial K}{\partial z_{j}^{*}}-\frac{\partial K}{\partial z_{i}}\left(\mathbf{C}^{-1}\right)_{i j} \frac{\partial F}{\partial z_{j}^{*}}\right], \\
& =\frac{1}{i \hbar}\{F, K\}_{\mathrm{GP}}
\end{aligned}
$$

where the complex generalized Poisson bracket (CGPB) [13] is defined as

$$
\begin{aligned}
\{A, B\}_{\mathrm{GP}} & =\left(\begin{array}{ll}
\frac{\partial A}{\partial z} & \frac{\partial A}{\partial z^{*}}
\end{array}\right)\left(\begin{array}{cc}
0 & \mathbf{C}^{-1} \\
-\left(\mathbf{C}^{-1}\right)^{t} & 0
\end{array}\right)\left(\begin{array}{c}
\frac{\partial B}{\partial z} \\
\frac{\partial B}{\partial z^{*}}
\end{array}\right), \\
& =\sum_{i=1}^{M} \sum_{j=1}^{M}\left[\frac{\partial A}{\partial z_{i}}\left(\mathbf{C}^{-1}\right)_{i j} \frac{\partial B}{\partial z_{j}^{*}}-\frac{\partial B}{\partial z_{i}}\left(\mathbf{C}^{-1}\right)_{i j} \frac{\partial A}{\partial z_{j}^{*}}\right] .
\end{aligned}
$$

\section{B. Second-class constraints}

In this section, we discuss the consistency conditions [10-12] for the constraints (4) to determine their Lagrange 
multipliers $\left\{\lambda_{j}\right\}_{j=1,2 L}$. The normalization constraint (3) will be considered in Secs. III and IV.

A basic requirement for the constraints in the TDVP is that the constraints should be preserved in time on the "trajectory" of the $\left\{z_{i}(t), z_{i}^{*}(t)\right\}_{i=1, M}$. This requirement is the same as the consistency condition in Dirac's constrained classical mechanics [11,12]. By using Eq. (15) without Eq. (3), the consistency conditions for the constraints (4) are written as

$$
\begin{aligned}
\frac{d g_{i}\left(z, z^{*}\right)}{d t}= & \frac{1}{i \hbar}\left\{g_{i}, H^{\prime}\right\}_{\mathrm{GP}}, \\
= & \frac{1}{i \hbar}\left\{g_{i}, H\right\}_{\mathrm{GP}}+\frac{1}{i \hbar} \sum_{j=1}^{2 L} \lambda_{j}\left\{g_{i}, g_{j}\right\}_{\mathrm{GP}}=0 \\
& (i=1, \ldots, 2 L) .
\end{aligned}
$$

Equations (17) can be considered as the inhomogeneous linear equations which determine the Lagrange multipliers $\left\{\lambda_{j}\right\}_{j=1,2 L}$. If we define a row vector $\mathbf{h}$,

$$
(\mathbf{h})_{i}=\left\{H, g_{i}\right\}_{\mathrm{GP}},
$$

and a skew-symmetric matrix $\mathbf{S}$,

$$
(\mathbf{S})_{j i}=\left\{g_{j}, g_{i}\right\}_{\mathrm{GP}},
$$

the inhomogeneous linear equations (17) are rewritten in the matrix form

$$
\mathbf{h}+\boldsymbol{\lambda} \mathbf{S}=\mathbf{0}
$$

Here we assume the nonsingularity of the skew-symmetric matrix $\mathbf{S}$. This assumption requires that the dimension of the
$\mathbf{S}$, or the number of the constraints in Eq. (4), should be even, $2 L$. The Lagrange multipliers can be uniquely determined from Eq. (20) as

$$
\lambda_{i}=-\sum_{j=1}^{2 L}(\mathbf{h})_{j}\left(\mathbf{S}^{-1}\right)_{j i}=-\sum_{j=1}^{2 L}\left\{H, g_{j}\right\}_{\mathrm{GP}}\left(\mathbf{S}^{-1}\right)_{j i} .
$$

In Dirac's constrained mechanics [11,12], the constraints whose Lagrange multipliers can be uniquely determined from their consistency conditions as the above are said to be the second-class constraints. The expectation value of the Hamiltonian (11) is written with Eq. (21) explicitly as

$$
H^{\prime}=\left\langle\Psi\left|\hat{H}^{\prime}\right| \Psi\right\rangle=H-\sum_{i=1}^{2 L} \sum_{j=1}^{2 L}\left\{H, g_{j}\right\}_{\mathrm{GP}}\left(\mathbf{S}^{-1}\right)_{j i} g_{i}
$$

\section{PARAMETRIZATION WITH COMPLETE LOCAL BASES}

If the local bases $\left\{\partial \Psi / \partial z_{i}\right\}_{i=1, M}$ in the parametrized wave functions (2) construct a complete system with the overlap matrix (14), we can have a resolution of the unit operator as

$$
\sum_{i, j=1}^{M}\left|\frac{\partial \Psi}{\partial z_{i}}\right\rangle\left(\mathbf{C}^{-1}\right)_{i j}\left\langle\frac{\partial \Psi}{\partial z_{j}}\right|=\hat{1} .
$$

The completeness of the local bases is discussed briefly in Appendix I. Using Eq. (23), we have for any Hermitian operators $\hat{A}$ and $\hat{B}$,

$$
\begin{aligned}
\{\langle\Psi|\hat{A}| \Psi\rangle,\langle\Psi|\hat{B}| \Psi\rangle\}_{\mathrm{GP}} & =\sum_{i, j=1}^{M}\left[\left\langle\Psi|\hat{A}| \frac{\partial \Psi}{\partial z_{i}}\right\rangle\left(\mathbf{C}^{-1}\right)_{i j}\left\langle\frac{\partial \Psi}{\partial z_{j}}|\hat{B}| \Psi\right\rangle-\left\langle\frac{\partial \Psi}{\partial z_{i}}|\hat{A}| \Psi\right\rangle\left[\left(\mathbf{C}^{-1}\right)^{t}\right]_{i j}\left\langle\Psi|\hat{B}| \frac{\partial \Psi}{\partial z_{j}}\right\rangle\right] \\
& =\left\langle\Psi\left|\hat{A}\left(\sum_{i, j=1}^{M}\left|\frac{\partial \Psi}{\partial z_{i}}\right\rangle\left(\mathbf{C}^{-1}\right)_{i j}\left\langle\frac{\partial \Psi}{\partial z_{j}}\right|\right) \hat{B}\right| \Psi\right\rangle-\left[\left\langle\Psi\left|\hat{A}\left(\sum_{i, j=1}^{M}\left|\frac{\partial \Psi}{\partial z_{i}}\right\rangle\left(\mathbf{C}^{-1}\right)_{i j}\left\langle\frac{\partial \Psi}{\partial z_{j}}\right|\right) \hat{B}\right| \Psi\right\rangle\right]^{*} \\
& =\langle\Psi|\hat{A} \hat{1} \hat{B}| \Psi\rangle-[\langle\Psi|\hat{A} \hat{1} \hat{B}| \Psi\rangle]^{*}, \\
& =\left\langle\Psi\left|[\hat{A}, \hat{B}]_{-}\right| \Psi\right\rangle .
\end{aligned}
$$

The CGPB of the expectation values of $\hat{A}$ and $\hat{B}$ has been proved to be equal to the expectation value of the commutator of those operators. Then, if the local bases construct a complete basis set, the classification of constraints with the CGPB becomes equivalent to that with the commutator of the corresponding operators [10].

\section{A. Norm of wave functions: First-class constraints}

For the complete local bases in Eq. (23), we consider the consistency condition for the normalization condition (3) to determine its Lagrange multiplier $\lambda_{0}$. By using Eqs. (15) and (24), the time variation of the normalization condition is calculated as

$$
\begin{aligned}
\frac{d g_{0}}{d t} & =\frac{1}{i \hbar}\{\langle\Psi|\hat{1}| \Psi\rangle, K\}_{\mathrm{GP}}, \\
& =\frac{1}{i \hbar}\left\{\langle\Psi|\hat{1}| \Psi\rangle,\left\langle\Psi\left|\hat{H}^{\prime}\right| \Psi\right\rangle\right\}_{\mathrm{GP}}+\frac{1}{i \hbar} \lambda_{0}\left\{g_{0}, g_{0}\right\}_{\mathrm{GP}}, \\
& =\frac{1}{i \hbar}\left\langle\Psi\left|\left[\hat{1}, \hat{H}^{\prime}\right]_{-}\right| \Psi\right\rangle=0 .
\end{aligned}
$$


This equation always holds because the constraining operator for the normalization is the unit operator which is commutable with any operators. Then we cannot determine uniquely the Lagrange multiplier $\lambda_{0}$ from the consistency requirement for the normalization condition. The constraints whose Lagrange multipliers cannot be uniquely determined from their consistency conditions are said to be the first-class constraints [11,12]. Then the normalization condition in the TDVP corresponds to the first-class constraint in Dirac's constrained mechanics. We fix here the Lagrange multiplier $\lambda_{0}$ directly to a real function $\Lambda$ as a gauge-fixing condition [10-12],

$$
\lambda_{0}(t)=\Lambda(t),
$$

where the real function $\Lambda$ can be selected arbitrarily.

\section{B. Constants of motion}

In this section, we summarize the constants of motion in our constrained system with the complete local bases in Eq. (23). First, the norm of the wave function is conserved in time as shown in Eq. (25). Using Eqs. (15) and (24), the time development of the expectation value of a Hermitian operator $\hat{\Omega}$ is calculated as

$$
\begin{aligned}
\frac{d\langle\Psi|\hat{\Omega}| \Psi\rangle}{d t}= & \frac{1}{i \hbar}\{\langle\Psi|\hat{\Omega}| \Psi\rangle, K\}_{\mathrm{GP}}, \\
= & \frac{1}{i \hbar}\left\{\langle\Psi|\hat{\Omega}| \Psi\rangle,\left\langle\Psi\left|\hat{H}^{\prime}\right| \Psi\right\rangle\right\}_{\mathrm{GP}}+\frac{1}{i \hbar} \Lambda(t) \\
& \times\left\{\langle\Psi|\hat{\Omega}| \Psi\rangle, g_{0}\right\}_{\mathrm{GP}}, \\
= & \frac{1}{i \hbar}\left\langle\Psi\left|\left[\hat{\Omega}, \hat{H}^{\prime}\right]_{-}\right| \Psi\right\rangle+\frac{1}{i \hbar} \Lambda(t)\left\langle\Psi\left|[\hat{\Omega}, \hat{1}]_{-}\right| \Psi\right\rangle, \\
= & \frac{1}{i \hbar}\left\langle\Psi\left|\left[\hat{\Omega}, \hat{H}^{\prime}\right]_{-}\right| \Psi\right\rangle .
\end{aligned}
$$

If the Hermitian operator $\hat{\Omega}$ is commutable with the Hamiltonian $\hat{H}^{\prime}$, the expectation value $\langle\Psi|\hat{\Omega}| \Psi\rangle$ will be a constant of motion with the normalization condition of the wave function. Next, in the case of $\hat{\Omega}=\hat{H}$ in particular, we have

$$
\left\{\langle\Psi|\hat{H}| \Psi\rangle,\left\langle\Psi\left|\hat{H}^{\prime}\right| \Psi\right\rangle\right\}_{\mathrm{GP}}=0,
$$

from only the antisymmetric property of the CGPB (16) without Eq. (24). Then the energy of the system is also conserved with the normalization condition of the wave function.

\section{PARAMETRIZATION WITH INCOMPLETE LOCAL BASES}

In this section, we consider approximate wave functions with limited variational parameters. Incomplete local bases with the limited variational parameters cannot afford the equivalence between the CGPB and the commutator of operators as shown in Eqs. (23) and (24). The first-class constraints and the constants of motion, classified with the com- mutator of the operators [10], should be generally reconsidered as the pseudo-second-class constraints in the approximate wave functions.

\section{A. Norm of wave functions}

\section{Parametrization 1: Pseudo-second-class constraints}

Here we consider approximate wave functions which are generally parametrized. We call the wave functions Parametrization 1 to distinguish them from a special parametrization scheme, which will be discussed in Sec. IV A 2.

We first relax the normalization condition (3) to the constant-norm condition of the wave function as

$$
N=\langle\Psi|\hat{1}| \Psi\rangle-N_{0}=0,
$$

where $N_{0}$ is a real constant given as the norm. By using Eq. (15), the time variation of the constraint (29) is calculated as

$$
\begin{aligned}
\frac{d N}{d t}=\frac{1}{i \hbar}\{N, K\}_{\mathrm{GP}} & =\frac{1}{i \hbar}\left\{N, H^{\prime}\right\}_{\mathrm{GP}}+\frac{1}{i \hbar} \lambda_{0}\{N, N\}_{\mathrm{GP}}, \\
& =\frac{1}{i \hbar}\left\{\langle\Psi|\hat{1}| \Psi\rangle,\left\langle\Psi\left|\hat{H}^{\prime}\right| \Psi\right\rangle\right\}_{\mathrm{GP}}, \\
& \neq\left\langle\Psi\left|\left[\hat{1}, \hat{H}^{\prime}\right]_{-}\right| \Psi\right\rangle=0 .
\end{aligned}
$$

For the lack of the completeness in Eq. (23), the norm of the wave function will generally change in time. We should constrain Eq. (30) to be zero as the consistency condition for the constant-norm condition. Consequently, the constraint (29) with the additional constraint (30) can be considered as a pair of the pseudo-second-class constraints. The new constraints can be included in Eq. (4) from the beginning as $(2 L+2)$ second-class constraints totally. For simplicity, however, we take only the components which are independent of the $2 L$ constraints in Eq. (4) in order not to disturb the constrained Hamiltonian which is already determined in Eq. (22). The component of the constraint (29), which is "orthogonal" to the already considered constraints (4), can be obtained by a Schmidt-like orthogonalization scheme using the CGPB as

$$
g_{0}=N-\sum_{i=1}^{2 L} \sum_{j=1}^{2 L}\left\{N, g_{j}\right\}_{\mathrm{GP}}\left(\mathbf{S}^{-1}\right)_{j i} g_{i}=0 .
$$

The consistency condition for Eq. (31) is

$$
\begin{aligned}
N^{\prime} & =\frac{d g_{0}}{d t}=\frac{1}{i \hbar}\left\{g_{0}, K\right\}_{\mathrm{GP}}=\frac{1}{i \hbar}\left\{g_{0}, H^{\prime}\right\}_{\mathrm{GP}}+\frac{1}{i \hbar} \lambda_{0}\left\{g_{0}, g_{0}\right\}_{\mathrm{GP}} \\
& =\frac{1}{i \hbar}\left\{g_{0}, H^{\prime}\right\}_{\mathrm{GP}}=0 .
\end{aligned}
$$

By orthogonalizing Eq. (32) to the already considered constraints (4) again, we have a new additional constraint

$$
h_{0}\left(z, z^{*}\right)=N^{\prime}-\sum_{i=1}^{2 L} \sum_{j=1}^{2 L}\left\{N^{\prime}, g_{j}\right\}_{\mathrm{GP}}\left(\mathbf{S}^{-1}\right)_{j i} g_{i}=0 .
$$

In fact, we have the "orthogonal" relations using the CGPB as 


$$
\left\{g_{0}, g_{i}\right\}_{\mathrm{GP}}=\left\{h_{0}, g_{i}\right\}_{\mathrm{GP}}=0 \quad(i=1, \ldots, 2 L) .
$$

The new Hamiltonian which includes the pseudo-secondclass constraints (31) and (33) is defined as

$$
K=H^{\prime}+\lambda_{0} g_{0}+\mu_{0} h_{0},
$$

where $\lambda_{0}$ and $\mu_{0}$ are the Lagrange multipliers for the new constraints. Using Eq. (15) with the Hamiltonian (35), we have linear equations

$$
\begin{aligned}
& \left\{g_{0}, H\right\}_{\mathrm{GP}}+\mu_{0}\left\{g_{0}, h_{0}\right\}_{\mathrm{GP}}=0, \\
& \left\{h_{0}, H\right\}_{\mathrm{GP}}+\lambda_{0}\left\{h_{0}, g_{0}\right\}_{\mathrm{GP}}=0,
\end{aligned}
$$

as the consistency conditions for the constraints (31) and (33). The Lagrange multipliers are determined like Eq. (21) for the second-class constraints,

$$
\begin{gathered}
\lambda_{0}=-\frac{\left\{h_{0}, H\right\}_{\mathrm{GP}}}{\left\{h_{0}, g_{0}\right\}_{\mathrm{GP}}} \\
\mu_{0}=-\frac{\left\{g_{0}, H\right\}_{\mathrm{GP}}}{\left\{g_{0}, h_{0}\right\}_{\mathrm{GP}}}=-\frac{i \hbar h_{0}}{\left\{g_{0}, h_{0}\right\}_{\mathrm{GP}}}=0 .
\end{gathered}
$$

Now we show a simple example of Parametrization 1. We consider a three-dimensional model space which is spanned by an orthonormal basis set $\left\{\chi_{i}(x)\right\}_{i=1,3}$. The completeness of the basis set in the model space is written as

$$
\sum_{i=1}^{3}\left|\chi_{i}\right\rangle\left\langle\chi_{i}\right|=\hat{1}
$$

We take a trial wave function parametrized with $\left\{z_{1}, z_{2}\right\}$ as

$$
\Psi\left(z_{1}, z_{2} ; x\right)=\chi_{3}(x)+z_{1}(t) \chi_{1}(x)+z_{2}(t) \chi_{2}(x) .
$$

For the wave function (41), the matrix $\mathbf{C}$ in Eq. (14) is calculated to be a two-dimensional unit matrix, and the local bases with the parameters $\left\{z_{1}, z_{2}\right\}$ become incomplete in the three-dimensional model space as

$$
\sum_{i, j=1}^{2}\left|\frac{\partial \Psi}{\partial z_{i}}\right\rangle\left(\mathbf{C}^{-1}\right)_{i j}\left\langle\frac{\partial \Psi}{\partial z_{j}}\left|=\sum_{i=1}^{2}\right| \chi_{i}\right\rangle\left\langle\chi_{i}|=\hat{1}-| \chi_{3}\right\rangle\left\langle\chi_{3}\right| \neq \hat{1} .
$$

The Hamiltonian is also calculated with the wave function (41) as

$$
\begin{aligned}
H= & \langle\Psi|\hat{H}| \Psi\rangle=H_{33}+z_{1}^{*} z_{1} H_{11}+z_{2}^{*} z_{2} H_{22} \\
& +z_{1}^{*} z_{1} H_{12}+z_{2}^{*} z_{1} H_{21}+z_{1} H_{31}+z_{2} H_{32}+z_{1}^{*} H_{13}+z_{2}^{*} H_{23},
\end{aligned}
$$

where $H_{i j}=\left\langle\chi_{i}|\hat{H}| \chi_{j}\right\rangle$. For simplicity, we consider only the constant-norm condition (29) as

$$
g_{0}\left(z, z^{*}\right)=\langle\Psi|\hat{1}| \Psi\rangle-N_{0}=1+z_{1}^{*} z_{1}+z_{2}^{*} z_{2}-N_{0}=0 .
$$

The additional constraint

$$
h_{0}=\frac{d g_{0}}{d t}=\frac{1}{i \hbar}\left\{g_{0}, H\right\}_{\mathrm{GP}}=-\frac{2}{\hbar} \operatorname{Im}\left[z_{1} H_{31}+z_{2} H_{32}\right]=0
$$

is considered as the partner of the pseudo-second-class constraint (44). The Lagrange multipliers are calculated with Eqs. (38) and (39) as

$$
\lambda_{0}=\frac{\operatorname{Re}\left[z_{1}\left(H_{11} H_{31}+H_{21} H_{32}\right)+z_{2}\left(H_{12} H_{31}+H_{22} H_{32}\right)\right]+\left|H_{31}\right|^{2}+\left|H_{32}\right|^{2}}{\operatorname{Re}\left[z_{1} H_{31}+z_{2} H_{32}\right]},
$$

and $\mu_{0}=0$. The EOM (13) for the parameters $\left\{z_{1}, z_{2}\right\}$ is written explicitly as

$$
\begin{aligned}
& \dot{z}_{1}=\frac{1}{i \hbar}\left[H_{13}+z_{1}\left(H_{11}+\lambda_{0}\right)+z_{2} H_{12}\right], \\
& \dot{z}_{2}=\frac{1}{i \hbar}\left[H_{23}+z_{2}\left(H_{22}+\lambda_{0}\right)+z_{1} H_{21}\right] .
\end{aligned}
$$

\section{Parametrization 2: First-class constraints}

In the preceding section we considered the normalization condition generally with the incomplete local bases. The constant-norm condition (29) has been calculated as the pseudo-second-class constraint. Here we take a different parameterization for approximate wave functions. This special parametrization, called here Parametrization 2, can keep the norm of the wave functions constant even if the local bases do not have the completeness in Eq. (23).

We consider a complex analytic parametrization of the trial wave function as

$$
\begin{aligned}
\Psi\left(z_{1}, z_{2}, \ldots, z_{M} ; x\right) & =z_{1} \Phi\left(z_{2}, \ldots, z_{M} ; x\right) \\
& =a_{1} e^{i \theta_{1}} \Phi\left(z_{2}, \ldots, z_{M} ; x\right),
\end{aligned}
$$

where an independent variational parameter $z_{1}$ is separated as the prefactor of the wave function $\Phi$. For the wave function (49), called Parametrization 2, the following equation always holds:

$$
\{\langle\Psi|\hat{1}| \Psi\rangle,\langle\Psi|\hat{B}| \Psi\rangle\}_{\mathrm{GP}}=0
$$

where $\hat{B}$ is an arbitrary operator. The proof of Eq. (50), which is given in Appendix II, does not need to use the 
completeness of the local bases in Eq. (23). If we take the operator $\hat{B}$ as the Hamiltonian $\hat{H}^{\prime}$, we have

$$
\left\{\langle\Psi|\hat{1}| \Psi\rangle,\left\langle\Psi\left|\hat{H}^{\prime}\right| \Psi\right\rangle\right\}_{\mathrm{GP}}=0 .
$$

This equation keeps the norm of the wave function (49) constant as discussed in Eq. (25) even without the completeness in Eq. (23). Therefore, the normalization condition (3) is kept as the first-class constraint for the approximate wave function (49), and the corresponding Lagrange multiplier $\lambda_{0}$ will be determined by the gauge-fixing condition (26) as discussed in Sec. III A.

Next we calculate $z_{1}(t)$ explicitly. The Lagrangian (5) for the wave function (49) is calculated as

$$
\begin{aligned}
L(z, z *)= & z_{1}^{*} z_{1}\left[\frac{i \hbar}{2}(\langle\Phi \mid \dot{\Phi}\rangle-\langle\dot{\Phi} \mid \Phi\rangle)-\left\langle\Phi\left|\hat{H}^{\prime}\right| \Phi\right\rangle\right] \\
& +\left[\frac{i \hbar}{2}\left(z_{1}^{*} \dot{z}_{1}-\dot{z}_{1}^{*} z_{1}\right)-\lambda_{0} z_{1}^{*} z_{1}\right]\langle\Phi \mid \Phi\rangle+\lambda_{0}, \\
= & z_{1}^{*} z_{1} L^{\prime}+\left[\frac{i \hbar}{2}\left(z_{1}^{*} \dot{z}_{1}-\dot{z}_{1}^{*} z_{1}\right)-\lambda_{0} z_{1}^{*} z_{1}\right]\langle\Phi \mid \Phi\rangle+\lambda_{0} .
\end{aligned}
$$

The Euler equations for $z_{1}^{*}(t)$ and $z_{1}(t)$ are

$$
\begin{aligned}
& i \hbar \dot{z}_{1}\langle\Phi \mid \Phi\rangle+z_{1}\left[L^{\prime}+\frac{i \hbar}{2}(\langle\Phi \mid \dot{\Phi}\rangle+\langle\dot{\Phi} \mid \Phi\rangle)-\lambda_{0}\langle\Phi \mid \Phi\rangle\right]=0, \\
& -i \hbar \dot{z}_{1}^{*}\langle\Phi \mid \Phi\rangle+z_{1}^{*}\left[L^{\prime}-\frac{i \hbar}{2}(\langle\Phi \mid \dot{\Phi}\rangle+\langle\dot{\Phi} \mid \Phi\rangle)-\lambda_{0}\langle\Phi \mid \Phi\rangle\right]=0 .
\end{aligned}
$$

Equations (53) and (54) are transformed to the EOM of the parameters $a_{1}$ and $\theta_{1}$ in Eq. (49) as

$$
\begin{gathered}
\dot{a}_{1}=-\frac{1}{2} a_{1} \frac{(\langle\Phi \mid \dot{\Phi}\rangle+\langle\dot{\Phi} \mid \Phi\rangle)}{\langle\Phi \mid \Phi\rangle}, \\
\dot{\theta}_{1}=\frac{1}{\hbar} \frac{\left[\frac{i \hbar}{2}(\langle\Phi \mid \dot{\Phi}\rangle-\langle\dot{\Phi} \mid \Phi\rangle)-\left\langle\Phi\left|\hat{H}^{\prime}\right| \Phi\right\rangle-\lambda_{0}\langle\Phi \mid \Phi\rangle\right]}{\langle\Phi \mid \Phi\rangle} .
\end{gathered}
$$

By using the normalization condition (3), the second-class conditions (4), and also the gauge-fixing condition (26), the variational parameter $z_{1}(t)$ is calculated finally as follows:

$$
z_{1}(t)=\frac{e^{i c}}{\sqrt{\langle\Phi \mid \Phi\rangle}} \exp \left[\frac{i}{\hbar} \int_{t_{0}}^{t} \frac{\left\langle\Phi\left|\frac{i \hbar}{2}\left(\vec{\partial}_{\tau}-\overleftarrow{\partial}_{\tau}\right)-\hat{H}-\Lambda\right| \Phi\right\rangle}{\langle\Phi \mid \Phi\rangle} d \tau\right] \text {, }
$$

where the real constant $c$ is determined from the initial phase of $z_{1}(t)$.

\section{B. Constants of motion as the pseudo-second-class constraints}

In this section, we summarize the constants of motion in approximate wave functions where Eq. (23) does not hold. First, the norm of the wave function can be conserved in both Parametrization 1 and Parametrization 2 as discussed in Secs. IV A 1 and IV A 2, respectively. As shown in Eq. (28), the expectation value of the Hamiltonian $\hat{H}$ is also conserved even without Eq. (24). Then the energy of the system is conserved.

Next we consider a Hermitian operator $\hat{\Omega}$ as a candidate of the constant motion which is commutable with the Hamiltonian $\hat{H}^{\prime}$ as

$$
\left[\hat{\Omega}, \hat{H}^{\prime}\right]_{-}=\hat{0} .
$$

We hope the expectation value

$$
\Omega=\langle\Psi|\hat{\Omega}| \Psi\rangle
$$

is a constant of motion as in Eq. (27). The already determined Hamiltonian with the constant-norm condition, Eq. (3) or Eq. (31), is written as

$$
K=H^{\prime}+\lambda_{0} g_{0},
$$

where $\lambda_{0}$ is determined by Eq. (38) with $\mu_{0}=0$ for Parametrization 1 . The Lagrange multiplier $\lambda_{0}$ is determined also for Parametrization 2 by the gauge-fixing condition $\Lambda$ (26). From Eq. (15), the time variation of $\Omega$ is calculated as

$$
\frac{d \Omega}{d t}=\frac{1}{i \hbar}\{\Omega, K\}_{\mathrm{GP}}=\frac{1}{i \hbar}\left\{\Omega, H^{\prime}\right\}_{\mathrm{GP}}+\frac{1}{i \hbar} \lambda_{0}\left\{\Omega, g_{0}\right\}_{\mathrm{GP}},
$$

where the right-hand side (rhs) of the equation is not always equal to zero because the rhs cannot be reduced to the commutator relations (58) and $[\hat{\Omega}, \hat{1}]_{-}=\hat{0}$ without Eq. (24). Then the expectation value $\Omega$ cannot be a constant of motion generally. Here we consider the Hermitian operator $\hat{\Omega}$ as an operator for the pseudo-second-class constraint. Considering the normalization, we introduce the new constraint as

$$
A=\langle\Psi|\hat{A}| \Psi\rangle=\langle\Psi|\hat{\Omega}-\Omega| \Psi\rangle=0 .
$$

Now we consider the wave function with Parametrization 1 as discussed in Sec. IV A 1. We extract only the component which is "orthogonal" to the already considered constraints by the Schmidt-like scheme as in Eq. (31),

$$
\begin{aligned}
g_{A}= & A-\sum_{i=1}^{2 L} \sum_{j=1}^{2 L}\left\{A, g_{j}\right\}_{\mathrm{GP}}\left(\mathbf{S}^{-1}\right)_{j i} g_{i}-\frac{\left\{A, h_{0}\right\}_{\mathrm{GP}}}{\left\{g_{0}, h_{0}\right\}_{\mathrm{GP}}} g_{0} \\
& -\frac{\left\{A, g_{0}\right\}_{\mathrm{GP}}}{\left\{h_{0}, g_{0}\right\}_{\mathrm{GP}}} h_{0}=0 .
\end{aligned}
$$

It is easy to show equations, $\left\{g_{A}, g_{i}\right\}_{\mathrm{GP}}=0,\left\{g_{A}, g_{0}\right\}_{\mathrm{GP}}=0$, and $\left\{g_{A}, h_{0}\right\}_{\mathrm{GP}}=0$. The consistency condition for Eq. (63) is calculated as 


$$
\begin{aligned}
A^{\prime} & =\frac{d g_{A}}{d t}=\frac{1}{i \hbar}\left\{g_{A}, K\right\}_{\mathrm{GP}}=\frac{1}{i \hbar}\left\{g_{A}, H^{\prime}\right\}_{\mathrm{GP}}+\frac{1}{i \hbar} \lambda_{0}\left\{g_{A}, g_{0}\right\}_{\mathrm{GP}} \\
& =\frac{1}{i \hbar}\left\{g_{A}, H^{\prime}\right\}_{\mathrm{GP}}=0 .
\end{aligned}
$$

By orthogonalizing Eq. (64) to the already considered constraints again, we have

$$
\begin{aligned}
g_{B}= & A^{\prime}-\sum_{i=1}^{2 L} \sum_{j=1}^{2 L}\left\{A^{\prime}, g_{j}\right\}_{\mathrm{GP}}\left(\mathbf{S}^{-1}\right)_{j i} g_{i}-\frac{\left\{A^{\prime}, h_{0}\right\}_{\mathrm{GP}}}{\left\{g_{0}, h_{0}\right\}_{\mathrm{GP}}} g_{0} \\
& -\frac{\left\{A^{\prime}, g_{0}\right\}_{\mathrm{GP}}}{\left\{h_{0}, g_{0}\right\}_{\mathrm{GP}}} h_{0}=0,
\end{aligned}
$$

and equations $\left\{g_{B}, g_{i}\right\}_{\mathrm{GP}}=0,\left\{g_{B}, g_{0}\right\}_{\mathrm{GP}}=0$, and $\left\{g_{B}, h_{0}\right\}_{\mathrm{GP}}=0$. The new Hamiltonian with the constraints (63) and (65) is defined as

$$
K=H^{\prime}+\lambda_{0} g_{0}+\lambda_{A} g_{A}+\mu_{B} g_{B} .
$$

From the consistency conditions for the pseudo-second-class constraints (63) and (65), we have linear equations as

$$
\begin{aligned}
& \left\{g_{A}, H\right\}_{\mathrm{GP}}+\mu_{B}\left\{g_{A}, g_{B}\right\}_{\mathrm{GP}}=0, \\
& \left\{g_{B}, H\right\}_{\mathrm{GP}}+\lambda_{A}\left\{g_{B}, g_{A}\right\}_{\mathrm{GP}}=0 .
\end{aligned}
$$

The Lagrange multipliers are determined like Eq. (21) for the second-class constraints,

$$
\begin{gathered}
\lambda_{A}=-\frac{\left\{g_{B}, H\right\}_{\mathrm{GP}}}{\left\{g_{B}, g_{A}\right\}_{\mathrm{GP}}}, \\
\mu_{B}=-\frac{\left\{g_{A}, H\right\}_{\mathrm{GP}}}{\left\{g_{A}, g_{B}\right\}_{\mathrm{GP}}}=-\frac{i \hbar g_{B}}{\left\{g_{A}, g_{B}\right\}_{\mathrm{GP}}}=0 .
\end{gathered}
$$

Finally, in the case of Parametrization 2 discussed in Sec. IV A 2, the trial wave function (49) always satisfies Eq. (51) and the norm of the wave function is conserved. Then we can drop all the terms of $g_{0}$ and $h_{0}$ from the above equations. The Lagrange multipliers $\lambda_{A}$ and $\mu_{B}$ in Parametrization 2 are the same as Eqs. (69) and (70), respectively.

\section{SUMMARY}

The TDVP with constraints is equivalent to solving the Schrödinger equation in restricted variational subspaces which are identified by some physical or artificial constraints. In this study, we have given the EOM with constraints for parametrized wave functions. If the local bases in the parametrized wave functions construct a complete basis set, the classification of the constraints with the CGPB becomes equivalent to that with the commutator of the corresponding operators [10]. The parametrization with such a complete local basis set can solve the Schrödinger equation exactly in the subspaces identified by the constraints. In the approximate wave functions, however, the local bases with limited variational parameters are generally incomplete and cannot afford the equivalence between the CGPB and the commutator of operators. Although the norm of the wave functions can be conserved by allocating an independent variational freedom to the prefactor of the wave functions, the first-class constraints and the constants of motion should be generally reconsidered as the pseudo-second-class constraints in the approximate wave functions.

\section{APPENDIX A: COMPLETENESS OF LOCAL BASES}

The completeness of the local bases $\left\{\partial \Psi / \partial z_{i}\right\}_{i=1, M}$ in Eq. (23) can be considered as follows. First we consider an orthonormal complete basis set $\left\{\chi_{i}\right\}_{i=1, M}$ in the Hilbert space with a resolution of the unit operator

$$
\sum_{i=1}^{M}\left|\chi_{i}\right\rangle\left\langle\chi_{i}\right|=\hat{1}
$$

We note that the bases $\left\{\chi_{i}\right\}_{i=1, M}$ are "nonlocal" because they do not depend on the variational parameters $\left\{z_{i}, z_{i}^{*}\right\}_{i=1, M}$. On the other hand, we can orthonormalize the local bases $\left\{\partial \Psi / \partial z_{i}\right\}_{i=1, M}$ by an adequate transformation as

$$
\xi_{i}\left(z, z^{*}\right)=\sum_{j=1}^{M} \frac{\partial \Psi(z)}{\partial z_{j}}\left[\mathbf{T}\left(z, z^{*}\right)\right]_{j i},
$$

where the transformation matrix $\mathbf{T}\left(z, z^{*}\right)$ should satisfy $\mathbf{T}^{\dagger} \mathbf{C T}=\mathbf{E}$ for the Hermitian overlap matrix $\mathbf{C}\left(z, z^{*}\right)$ in Eq. (14). If the orthonormalized but nonanalytical local bases $\left\{\xi_{i}\left(z, z^{*}\right)\right\}_{i=1, M}$ can be represented by the unitary transformation of the "nonlocal" bases $\left\{\chi_{i}\right\}_{i=1, M}$ as

$$
\xi_{i}\left(z, z^{*}\right)=\sum_{j=1}^{M} \chi_{j}\left[\mathbf{U}\left(z, z^{*}\right)\right]_{j i},
$$

we can show the completeness of the local bases $\left\{\partial \Psi / \partial z_{i}\right\}_{i=1, M}$ as

$$
\begin{aligned}
& \sum_{i, j=1}^{M}\left|\frac{\partial \Psi}{\partial z_{i}}\right\rangle\left(\mathbf{C}^{-1}\right)_{i j}\left\langle\frac{\partial \Psi}{\partial z_{j}}\right| \\
& =\sum_{i, j=1}^{M} \sum_{k, l=1}^{M}\left|\xi_{k}\right\rangle\left(\mathbf{T}^{-1}\right)_{k i}\left(\mathbf{C}^{-1}\right)_{i j}\left[\left(\mathbf{T}^{-1}\right)^{\dagger}\right]_{j l}\left\langle\xi_{l}\right|, \\
& =\sum_{k, l=1}^{M}\left|\xi_{k}\right\rangle\left[\left(\mathbf{T}^{\dagger} \mathbf{C} \mathbf{T}\right)^{-1}\right]_{k l}\left\langle\xi_{l}\left|=\sum_{i=1}^{M}\right| \xi_{i}\right\rangle\left\langle\xi_{i}\right|, \\
& =\sum_{i=1}^{M} \sum_{j=1}^{M} \sum_{k=1}^{M}\left|\chi_{j}\right\rangle(\mathbf{U})_{j i}\left(\mathbf{U}^{\dagger}\right)_{i k}\left\langle\chi_{k}\left|=\sum_{i=1}^{M}\right| \chi_{i}\right\rangle\left\langle\chi_{i}\right|, \\
& =\hat{1} .
\end{aligned}
$$

The unitarity of the "local" transformation $\mathbf{U}\left(z, z^{*}\right)$ in Eq. (A3) ensures the completeness of the local bases $\left\{\partial \Psi / \partial z_{i}\right\}_{i=1, M}$.

\section{APPENDIX B: PROOF OF EQ. (50)}

To prove Eq. (50), we first calculate the following terms for a real function $A$ as 


$$
I_{i}=\sum_{j=1}^{M} \frac{\partial A}{\partial z_{j}}\left(\mathbf{C}^{-1}\right)_{j i}=\left[\sum_{j=1}^{M}\left(\mathbf{C}^{-1}\right)_{i j} \frac{\partial A}{\partial z_{j}^{*}}\right]^{*} \quad(i=1, \ldots, M) .
$$

By using the terms $I_{i}$, the CGPB (16) is written as

$$
\{A, B\}_{\mathrm{GP}}=\sum_{i=1}^{M}\left[I_{i} \frac{\partial B}{\partial z_{i}^{*}}-\frac{\partial B}{\partial z_{i}} I_{i}^{*}\right] .
$$

Multiplying Eq. (B1) by the overlap matrix C, we have

$$
\sum_{i=1}^{M} I_{i}(\mathbf{C})_{i j}=\frac{\partial A}{\partial z_{j}} \quad(j=1, \ldots, M) .
$$

For the wave function (49) in Parametrization 2, the local bases are calculated as

$$
\begin{gathered}
\frac{\partial \Psi}{\partial z_{1}}=\Phi \\
\frac{\partial \Psi}{\partial z_{i}}=z_{1} \frac{\partial \Phi}{\partial z_{i}} \equiv z_{1} \Phi_{i} \quad(i=2, \ldots, M) .
\end{gathered}
$$

The overlap matrix $\mathbf{C}$ in Eq. (14) between the local bases (B4) and (B5) is calculated as

$$
\mathbf{C}=\left(\begin{array}{cccc}
\langle\Phi \mid \Phi\rangle & z_{1}\left\langle\Phi \mid \Phi_{2}\right\rangle & \ldots & z_{1}\left\langle\Phi \mid \Phi_{M}\right\rangle \\
z_{1}^{*}\left\langle\Phi_{2} \mid \Phi\right\rangle & \ldots & \ldots & \ldots \\
\ldots & \ldots & z_{1}^{*} z_{1}\left\langle\Phi_{i} \mid \Phi_{j}\right\rangle & \ldots \\
z_{1}^{*}\left\langle\Phi_{M} \mid \Phi\right\rangle & \ldots & \ldots & \ldots
\end{array}\right) .
$$

If we take the norm of the wave function (49) as the real function $A$ in Eq. (B1), we have

$$
\begin{gathered}
A=\langle\Psi|\hat{1}| \Psi\rangle=z_{1}^{*} z_{1}\langle\Phi \mid \Phi\rangle, \\
\frac{\partial A}{\partial z_{1}}=z_{1}^{*}\langle\Phi \mid \Phi\rangle, \\
\frac{\partial A}{\partial z_{i}}=z_{1}^{*} z_{1}\left\langle\Phi \mid \Phi_{i}\right\rangle, \text { and c.c. }
\end{gathered}
$$

By calculating Eq. (B3) with Eqs. (B6)-(B8), we have, for $j=1$,

$$
I_{1}\langle\Phi \mid \Phi\rangle+z_{1}^{*} \sum_{i=2}^{M} I_{i}\left\langle\Phi_{i} \mid \Phi\right\rangle=z_{1}^{*}\langle\Phi \mid \Phi\rangle,
$$

and for $j \geqslant 2$,

$$
I_{1} z_{1}\left\langle\Phi \mid \Phi_{j}\right\rangle+z_{1}^{*} z_{1} \sum_{i=2}^{M} I_{i}\left\langle\Phi_{i} \mid \Phi_{j}\right\rangle=z_{1}^{*} z_{1}\left\langle\Phi \mid \Phi_{j}\right\rangle .
$$

The elimination of $I_{1}$ from Eqs. (B9) and (B10) leads to the homogeneous linear equations for $I_{i}(i \geqslant 2)$,

$$
\sum_{i=2}^{M} I_{i}\left[\langle\Phi \mid \Phi\rangle\left\langle\Phi_{i} \mid \Phi_{j}\right\rangle-\left\langle\Phi_{i} \mid \Phi\right\rangle\left\langle\Phi \mid \Phi_{j}\right\rangle\right]=0 \quad(j=2, \ldots, M) .
$$

By introducing the matrix $\mathbf{D}$ as

$$
(\mathbf{D})_{i j}=\langle\Phi \mid \Phi\rangle\left\langle\Phi_{i} \mid \Phi_{j}\right\rangle-\left\langle\Phi_{i} \mid \Phi\right\rangle\left\langle\Phi \mid \Phi_{j}\right\rangle \quad(i, j=2, \ldots, M),
$$

we can rewrite Eq. (B11) as

$$
\sum_{i=2}^{M} I_{i}(\mathbf{D})_{i j}=0 \quad(j=2, \ldots, M) .
$$

On the other hand, the determinant of the matrix $\mathbf{C}$ (B6) is calculated by expanding in the first column as

$$
|\mathbf{C}|=\langle\Phi \mid \Phi\rangle^{-(M-2)}\left(z_{1}^{*} z_{1}\right)^{(M-1)}|\mathbf{D}| .
$$

If we can assume $|\mathbf{C}| \neq 0$ as the nonsingularity of the local bases (B4) and (B5), Eq. (B14) leads directly to

$$
|\mathbf{D}| \neq 0 \text {. }
$$

From Eq. (B15), we have only the trivial solutions for Eq. (B13) as

$$
I_{i}=0 \quad(i=2, \ldots, M) .
$$

By substituting Eq. (B16) into Eq. (B9), we have

$$
I_{1}=z_{1}^{*} \text {. }
$$

Finally, for any operator $\hat{B}$, the CGPB is calculated through Eq. (B2) as

$$
\begin{aligned}
\{\langle\Psi|\hat{1}| \Psi\rangle,\langle\Psi|\hat{B}| \Psi\rangle\}_{\mathrm{GP}}= & z_{1}^{*} \frac{\partial\left(z_{1}^{*} z_{1}\langle\Phi|\hat{B}| \Phi\rangle\right)}{\partial z_{1}^{*}} \\
& -\frac{\partial\left(z_{1}^{*} z_{1}\langle\Phi|\hat{B}| \Phi\rangle\right)}{\partial z_{1}} z_{1}, \\
& =0 .
\end{aligned}
$$

[1] P. A. M. Dirac, Proc. R. Soc. London, Ser. A 112, 673 (1926).

[2] P. A. M. Dirac, Proc. Cambridge Philos. Soc. 26, 376 (1930).

[3] J. Frenkel, Wave Mechanics, Advanced General Theory (Clarendon, Oxford, 1934).

[4] P.-O. Löwdin and P. K. Mukherjee, Chem. Phys. Lett. 14, 1
(1972).

[5] A. D. McLachlan, Mol. Phys. 8, 39 (1964).

[6] P. M. Morse and H. Feshbach, Methods of Theoretical Physics (McGraw-Hill, New York, 1953).

[7] E. Deumens, A. Diz, R. Longo, and Y. Öhrn, Rev. Mod. Phys. 
66, 917 (1994), and references therein.

[8] I. M. Gelfand and S. V. Fomin, Calculus of Variations (Prentice-Hall, Englewood Cliffs, NJ, 1963).

[9] L. E. Elsgolc, Calculus of Variations (Pergamon, Oxford, 1962).

[10] K. Ohta, Chem. Phys. Lett. 329, 248 (2000).
[11] P. A. M. Dirac, Can. J. Math. 2, 129 (1950).

[12] P. A. M. Dirac, Lectures on Quantum Mechanics (Yeshiva University Press, New York, 1964).

[13] P. Kramer and M. Saraceno, Geometry of the Time-Dependent Variational Principle in Quantum Mechanics (Springer, New York, 1981). 